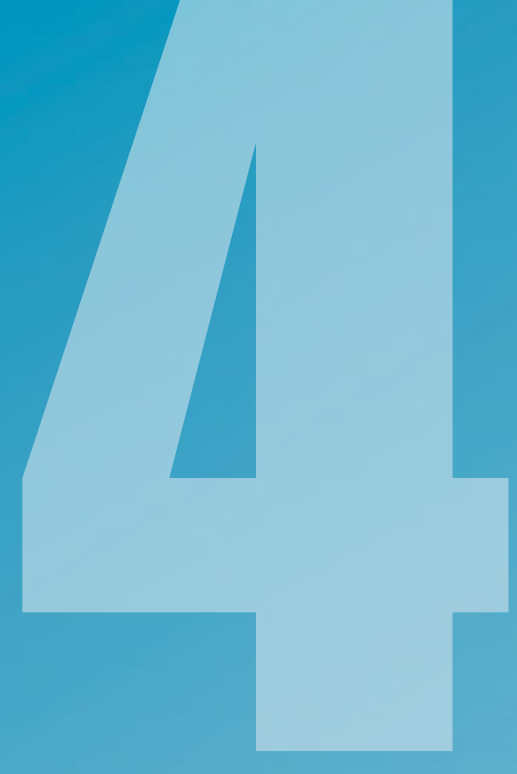

ÁMBITOS

REVISTA

INTERNACIONAL

DE COMUNICACIÓN

$N^{\circ} 46$

EDICIÓN OTOÑO

2019

ISSN: 1139-1979

E-ISSN: 1988-5733

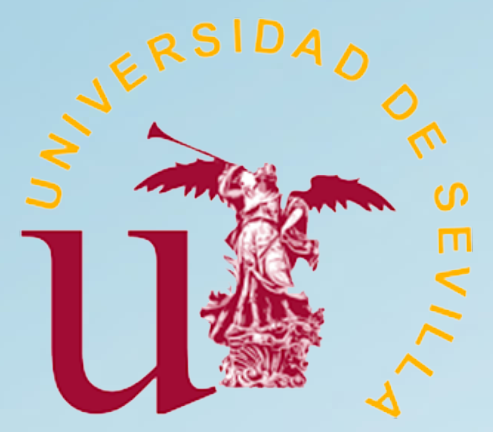




\section{ÍNDICE}

MONOGRAFICOS MONOGRAPHS

Presentación Monográfico. Comunicación emergente. Experiencias para el cambio social

Dra. Nereida López Vidales

Los formatos de televisión más consumidos por los jóvenes: telerrealidad y empoderamiento de la audiencia

Most consumed televisión formats by young people: real TV and the empowerment of the audience Nereida López Vidales, Leire Gómez Rubio, Elena Medina de la Viña

Nuevas herramientas, viejas costumbres El Contenido Generado por los Usuarios sobre el cambio climático en YouTube

New tools, old habits User Generated Content about climate change on YouTube

David Vicente Torrico

Tratamiento periodístico de personas LGTBIQ+ refugiadas: estudio de caso sobre Pride Barcelona 2018

Journalistic treatment of LGTBIQ+ refugees: case study on Pride Barcelona 2018

Hadriel Theodoro, Amparo Huertas Bailén

Tiempo Muerto, estudio de caso de un proyecto transmediático para la consecución de competencias universitarias

Tiempo Muerto, case study of a transmediatic project for the achievement of university competences Jose L. Carreño Villada, Miguel Ángel Díaz Monsalvo

Los universitarios millennials: uso de redes sociales y relación con las marcas

University Millennials: use of social media and engage with brands

Pedro Pablo Marín Dueñas, Esther Simancas González

Ali Ferzat: De la caricatura comunicativa en papel a la caricatura activista en los medios digitales

Ali Ferzat: From political cartoons in papers to political cartoons in digital media

Salud Adelaida Flores Borjabad

Direito à informação e literacia midiática: Reflexões sobre a questão do acesso

Right to information and media literacy: Reflections on the question of acce

Christiane Delmondes Versuti 
Eficacia de los influencers como recurso publicitario en la estrategia de los anunciantes locales Efficacy of influencers as an advertising resource in the strategy of local advertisers

Isabel Iniesta-Alemán

As redes sociais como ferramenta de marketing em instituições de ensino superior no Brasil Social networks as a marketing tool in higher education institutions in Brazil

Alcino Ricoy JR, Rogério Eduardo Rodrigues Bazi

Estudio sobre la conceptualización y el tratamiento informativo de la violencia de género en la prensa digital cubana

Study about the conceptualization and information treatment of gender violence in the Cuban digital press

Regla Ismaray Cabreja Piedra, Karina Escalona Peña

\section{ARTÍCULOS ARTICLES}

Uso de Twitter durante los debates electorales televisados en los comicios andaluces de 2018 The use of Twitter during the televised electoral debates in the 2018 Andalusian elections Julia Fontenla Pedreira, Erika Conde Vázquez, Carmen Máiz Bar

Quem averigua as notícias, os algoritmos ou jornalistas? A lógica crítica de C. S.

Peirce como processo de identificação de uma Fake News

Who checks the news, algorithms or journalists? The critical logic of C. S. Peirce as a

process for identifying a Fake News

Adelino de Castro Oliveira Simões Gala, Vania Baldi, Universidad de Aveiro

\section{RESEÑAS REVIEWS}

Nuevo ecosistema comunicativo digital: El consumidor

Juan Carlos Figuereo Benítez

Corpus toponímic de Beniarrés

Bianca Sánchez-Gutiérrez

The Future Computed. La inteligencia artificial y su papel en la sociedad y Pulsa actualizar. La aventura de redescubrir el alma de Microsoft y concebir un futuro mejor para todos 


\title{
Estudio sobre la conceptualización y el tratamiento informativo de la violencia de género en la prensa digital cubana
}

\author{
Study about the conceptualization and information treatment of \\ gender violence in the Cuban digital press
}

\author{
Regla Ismaray Cabreja Piedra, Universidad de Sevilla, \\ Calle Castilla 141, Sevilla \\ ismapiedra17@gmail.com | Orcid: https://orcid.org/0000-0002-5969-3222 \\ Karina Escalona Peña, Universidad de Sevilla, Universidad Central del Ecuador, \\ Bolivia Oe7-132 y Eustorgio Salgado, Quito \\ karina.escalona83@gmail.com | Orcid: https://orcid.org/0000-0002-1524-285X
}

DOI: http://dx.doi.org/10.12795/Ambitos.2019.i46.11

\begin{abstract}
Resumen
Los medios, como principales agentes sociales del cambio, son fundamentales para guiar la lucha feminista por la igualdad y contra la violencia de género. Bajo esta premisa, se plantea el propósito de analizar la conceptualización y el tratamiento informativo de la violencia de género en la prensa digital. Se toma a Cuba como contexto específico, en donde el camino hacia la desnaturalización de mitos, prejuicios y estereotipos que legitiman la violencia de género todavía es una carrera pendiente.

El problema de investigación encuentra como método para su estudio el análisis de contenido cualitativo, el que provee las herramientas necesarias para la descripción e
\end{abstract}


interpretación de los textos producidos por la prensa digital. Este análisis se realiza desde la información publicada en medios digitales con énfasis en las noticias sobre violencia de género en Cuba, tomando como período de tiempo el comprendido de enero a diciembre de 2017. Para mayor riqueza en el análisis fueron seleccionados distintos tipos de medios, dentro ellos se encuentran los oficiales, de propiedad estatal y medios independientes, de propiedad no estatal.

Los resultados expuestos propician el entendimiento sobre el tratamiento que ha recibido el tema, además de que contribuyen a la determinación de los principales elementos que han pautado la conceptualización del problema en estas agendas. Del análisis deriva la evidencia de una muy limitada cobertura, con sesgos informativos y un estado de tratamiento de bajo perfil en muchas ocasiones, que manifiestan la necesidad de darle prioridad a este problema social de gran escala.

\section{Abstract}

The media, as the main social agents of change, are fundamental in guiding the feminist struggle for equality and against gender violence. Under this premise, the purpose is to analyze the conceptualization and the informative treatment of gender violence in the digital press. Cuba is taken as a specific context, where the path towards the denaturalization of myths, prejudices and stereotypes that legitimize gender violence is still a pending race.

The problem of investigation finds as a method for its study the analysis of qualitative content, which provides the necessary tools for the description and interpretation of the texts produced by the digital press. This analysis is carried out from information published in digital media with an emphasis on news about gender violence in Cuba, taking as a period the period from January to December 2017. For greater wealth in the analysis, different types of media were selected, inside them are the state-owned and independent media officers, non-state property.

The exposed results propitiate the understanding on the treatment that has received the subject, besides that they contribute to the determination of the main elements that have guided the conceptualization of the problem in these agendas. From the analysis derives the evidence of a very limited coverage, with informative biases and a state of treatment of low profile in many occasions, which manifest the need to give priority to this social problem of large scale.

Palabras clave: Violencia de género, Feminismo, Análisis cualitativo, Medios de Comunicación, Cuba

Keywords: Gender violence, Feminism, Qualitative analysis, Communication Media, Cuba 


\section{INTRODUCCIÓN}

Los medios de comunicación en general y el periodismo en particular constituyen una herramienta eficaz para la educación de los públicos. Su apego a construcciones culturales tradicionales, lejos de contribuir al diálogo con respecto a la igualdad de género, enfatiza las bases del patriarcado y ayuda a legitimar las diferencias entre hombres y mujeres.

La prensa es uno de los ámbitos que ha contribuido a fortalecer y legitimar la sumisión y opresión por razones de género, apelando a un concepto de igualdad sesgado que en realidad queda definido a conveniencia de quienes lo dictan. "Los medios pueden visibilizar u ocultar los problemas sociales (agenda setting) pero una vez que deciden visualizarlos pueden hacerlo de diversas maneras (jerarquizando su importancia y decidiendo sobre enfoques, conceptualizaciones y terminología)" (Zurbano \& García, 2017, p. 83).

El diarismo y la prevalencia de noticias sobre hechos aislados de violencia de género van en detrimento de hacer visible su verdadero impacto en la sociedad, cubriendo estereotipos que constituyen mecanismos de dominación perfeccionados a través de la historia y validados por los presupuestos que el patriarcado naturaliza.

La violencia contra las mujeres desde la perspectiva de género ha sido asumida como un serio problema social derivado de las desiguales relaciones de poder en las interacciones entre hombres y mujeres a nivel individual, grupal, explicado a partir de las asignaciones socioculturales a los cuerpos sexuados, y que se ha legitimado históricamente a través de la organización patriarcal de las sociedades.

Pero la cultura patriarcal no se reproduce por generación espontánea. Se educa y transmite de una generación a otra a través de la socialización diferente, que prepara a hombres y mujeres en subculturas diferentes. Unos para el ejercicio del poder y otras para la subordinación y la dependencia (Proveyer, 2005, p. 78).

Es por ello que la trascendencia de visibilizar el problema desde sus causas va más allá de hacer notar dónde están las manifestaciones de la violencia, sino de comenzar a romper el ciclo de formación y reproducción de modelos de hombres y mujeres marcados por las asignaciones de género. Entre las mayores dificultades para asumir este reto se encuentra la legitimación social de mitos y estereotipos, validados por la cultura patriarcal, que colabora con la reproducción de la subordinación femenina.

Se trata de un problema global y público, por lo que cualquier esfuerzo será menor si desde las agendas de los gobiernos no se tienen claras sus dimensiones y no se impulsan las acciones necesarias para combatirlo. "Conceptualizar es politizar", al decir de Celia Amorós (2005, p. 15), por tanto, la conceptualización de la violencia es clave 
en este sentido y a nivel teórico ha tenido una evolución importante, lo que ha llevado a los gobiernos a problematizar sobre las definiciones que se han estado manejando en los ámbitos mediáticos, jurídicos, sanitarios, educativos, etc.

El objetivo general es analizar la conceptualización y el tratamiento informativo de la violencia de género en los discursos mediáticos digitales en Cuba. Como objetivos específicos se presentan: identificar las definiciones que sobre violencia de género se han producido en el ámbito mediático digital en el contexto de Cuba; caracterizar el tratamiento de la violencia de género en medios digitales enfocados en la realidad cubana; identificar las limitaciones en la conceptualización y el tratamiento mediático digital de la violencia de género en Cuba.

El tema constituye un enfoque de análisis a la gravedad del problema. "El periodismo debe ser un aliado en la lucha por la erradicación de las violencias" (Zurbano y García, 2016, p. 4). En las agendas mediáticas cubanas se encuentra enraizada una ideología androcéntrica dominante que se invisibiliza en la producción del contenido, "la prensa se constituye en ámbito donde cohabitan las nuevas y viejas concepciones sobre lo masculino y lo femenino" (Moya, 2010, p. 98).

En Cuba, los actos violentos contra las mujeres reciben una prácticamente nula cobertura mediática, con lo cual se elude un problema social latente. "La representación sesgada, discriminatoria, binaria y excluyente de ambos géneros, así como la escasa presencia de las mujeres en el discurso mediático, son también formas de violencia simbólica, relacionadas con la violencia de género" (Alonso, 2018).

Por otro lado, no existe un referente de Ley que se encargue de prevenir y sancionar la violencia de género en el país, el Código Penal, vigente desde 1988, tampoco la tipifica como delito y por lo tanto, se carece de un aparato legal que paute la conceptualización del problema, lo que deriva en que los medios no tengan un referente establecido.

Pequeños pasos que se van dando en la visibilidad de estadísticas, merece la pena destacar las anunciadas en abril de este año en un Informe nacional ofrecido a la Comisión Económica para América Latina y el Caribe (CEPAL), sobre cómo se afronta la Agenda 2030 para el Desarrollo Sostenible. En el mismo se ofrecen datos sobre cómo se comportó la tasa de femicidios ${ }^{1}$ en 2016.

"El número de muerte ocasionadas por su pareja o expareja han disminuido entre el 2013 y 2016 en un 33 por ciento. En este último año la tasa de femicidios fue de 0,99 por 100000 habitantes de la población femenina de 15 años y más" (Informe voluntario de Cuba, 2019, p. 64). 
Aunque resulta una novedad que se den a conocer estas cifras en un país en el que poco se conoce de casos específicos de agresiones contra la mujer, su publicación no es suficiente si tenemos en cuenta que: no es un dato que ha sido argumentado de forma sistemática; no se profundiza en cómo se determinaron estas cifras; se utiliza una categoría que ni siquiera se encuentra amparada en el marco regulatorio jurídicopenal del país.

El hecho de que no se cuente con una Ley contra la violencia de género limita mucho las actuaciones en este sentido. "Visibilizar en una ley que existe el delito de violencia de género, provocado por la prácticas del patriarcado, permitiría actuar de un modo más efectivo contra quienes cometen esos actos y también sobre las causas que los provocan", asegura la psicóloga Mareelén Díaz Tenorio (Más, 2019).

Se hace preciso partir de definiciones sobre la violencia que no omita sus dimensiones y que declare tal cual sus implicaciones con claridad conceptual. "La tecnificación del problema, responde a un concepto simplificado de las violencias contras las mujeres (VVCMM). Un concepto anclado en la afectividad y la manifestación física del dominio ya que sólo las esposas y/o novias son susceptibles de ser víctimas y con las herramientas de medición actuales las únicas violencias detectables son las físicas de carácter grave" (Zurbano, 2015, p. 49).

La deficiente conceptualización que dan los medios de la violencia de género constituye un problema grave pues atenta contra la educación en torno al mismo, favorece la reproducción de estereotipos de género, ayuda a sustentar precisamente lo que el movimiento feminista quiere desmontar, la creencia de que es un problema aislado, que sus agresores son hombres con problemas psicológicos y que las víctimas 'se lo buscaron'. "Cada vez se hace más evidente la necesidad de ampliar y complejizar el concepto de violencia contra las mujeres con el fin de aumentar y mejorar la visibilidad de este fenómeno en todas sus consecuencias y de hacer más eficaces las medidas sancionadoras y de protección implementadas en los últimos años" (Zurbano, 2015, p. $52)$.

Para el análisis se toma de la información publicada en la prensa digital por ser también una fuente legitimadora de discursos machistas. Este tema ha sido llevado a discusión por organismos internacionales y así lo ha puesto en evidencia el documento de Resultados de la Sesión Especial de Naciones Unidas, al plantear que "las imágenes negativas, violentas o degradantes de la mujer, incluida la pornografía, y sus descripciones estereotipadas han aumentado en diferentes formas, recurriendo a nuevas tecnologías de la información en algunos casos, y los prejuicios contra la mujer siguen existiendo en los medios de difusión" (Informe del Comité Especial Plenario, 2000, p. 19). 
En Cuba, los contenidos de los medios digitales oficiales están igualmente normados que lo medios tradicionales por el Comité Central del Partido. El Doctor en Ciencias de la Comunicación, Julio García Luis, explica que la política informativa, en el contexto cubano, es la traducción de la agenda del sistema político a la prensa. Se puede decir que existe una estrategia informativa planteada en grandes líneas, pero la experiencia y creatividad profesional -de periodistas y directivos, en decisiones unilaterales o conjuntas-, indican cómo debe ser el seguimiento de cualquiera de estos tópicos (2011, p. $68)$.

Las limitaciones principales de la producción en medios digitales se acompañan de las mismas que caracterizan a los medios de comunicación tradicionales. El desarrollo de la web cubana ha corrido según urgencias, pero no se conocen políticas sobre esta, ni sistematización tampoco, por lo que la necesidad de una política de comunicación para la prensa en Cuba, ha constituido la principal causa de los problemas que caracterizan al sistema de medios.

Existe un ordenamiento de agenda bajo estricto control utilizado a discreción que limita el tratamiento de algunos temas. Ciertos matices instrumentales en la función de los medios y el predominio de la regulación externa, derivado de las presiones y peligros que encara el país, se reflejan también en las determinaciones internas de la prensa en cuanto a estructuras, atribuciones de poder, rutinas productivas, culturas profesionales y modelos de valor. La comunicación pública en Cuba es directamente funcional a la ideología hegemónica y al sistema político. Son algunas de las conclusiones a las que llega Julio García sobre los elementos definitorios de los medios de comunicación en Cuba (2013, p. 110).

El espectro mediático digital en el país se encuentra conformado por medios de propiedad estatal, reconocidos como oficiales y medios de propiedad no estatal, reconocidos como independientes, dentro de los que hay algunos con agendas marcadamente opositoras al sistema político del país, como es el caso de Martí Noticias, CiberCuba, etc. No existe un consenso en torno a las distintas tipologías de medios en Cuba ni sobre las formas de propiedad de estos, así como tampoco existe una Ley de Prensa o de Medios que organice las prácticas profesionales en este sector. La nueva Constitución de la República de Cuba, aprobada a finales de 2018 por la Asamblea Nacional del Poder Popular y que entró en vigor el 10 de abril de 2019, sustituyendo a la Constitución de 1976, tampoco reconoce los diversos tipos de medios que existen. En su Artículo 55, solo reconoce los de propiedad estatal, al decir que "los medios fundamentales de comunicación social, en cualquiera de sus manifestaciones y soportes, son de propiedad socialista de todo el pueblo o de las organizaciones políticas, sociales y de masas; y no pueden ser objeto de otro tipo de propiedad" (Constitución de la República de Cuba, 2019, p. 77). 
Todavía existen normativas ancestrales que precisan adaptarse a la nueva realidad que caracteriza al país. Un paso favorable en este sentido es la nueva Constitución, de la misma se cita en el Título III, artículo 43, que el Estado protege a las mujeres de "la violencia de género en cualquiera de sus manifestaciones y espacios, y crea los mecanismos institucionales y legales para ello" (íbidem). Sobre esto Díaz apunta que "solo que lo que realmente se va a hacer está por venir (...) Hay urgencia, ya nos sentimos atrasados jurídicamente. Ojalá nos sirva para apretar el paso, porque la legislación de hoy está muy lejos de nuestra realidad" (Más, 2019).

Es un hecho que "la violencia de género está presente aún en la sociedad cubana, con todas las implicaciones que se derivan de esta problemática para las mujeres en cualquier parte del mundo; sus manifestaciones adquieren los mismos matices, desde un silencio omisor hasta la muerte, porque la estructura social patriarcal, aunque menos monolítica debido a los cambios operados a nivel social, sigue sirviendo de sostén a la dominación masculina" (Proveyer, 2014).

Nos aproximamos a las conceptualizaciones con el interés de comprender con rigor científico cómo los contenidos producidos por la presa digital cubana construyen versiones distintas sobre la realidad, otorgándole matices diversos que implican, por tanto, construcciones sociales diversas. Los medios de comunicación en Cuba muchas veces reproducen el sexismo manifiesto de una cultura patriarcal que normaliza estos comportamientos. "Si bien, es cierto, que los medios son muchas veces invocados para denunciar las representaciones que hacen de lo femenino y lo masculino, por lo general se quedan en apreciaciones vivenciales, en hechos aislados y en no escasas ocasiones desde una visión funcionalista" (Moya, 2010, p. 104).

Como hipótesis se parte del hecho de que desde los discursos mediáticos digitales se producen conceptualizaciones limitadas sobre la violencia de género ya que por un lado, tienden a estar asociadas a contenidos que abordan el fenómeno de forma eventual, por lo cual no reflejan concretamente el estado actual de esta problemática en el país. Esta situación encuentra un agravante en la baja prioridad que le atribuyen los medios digitales, expresado en la muy limitada presencia de textos, de estadísticas informativas, así como de historias de vida que reflejen el arraigo social del problema.

La investigación encuentra como método para su estudio el análisis de contenido cualitativo, el que se realiza desde la información publicada en medios digitales de referencia en Cuba con énfasis en las noticias sobre violencia de género. Piñuel define este método de análisis como "el conjunto de instrumentos interpretativos de productos comunicativos (mensajes, textos, discursos) y que basados en técnicas de medida cualitativas (lógicas sustentadas en la combinación de categorías) tienen por objeto elaborar y procesar datos relevantes sobre las condiciones mismas en que se han 
producido aquellos textos, o sobre las condiciones que pueden darse para su empleo posterior" (2002, p. 7).

Se ha utilizado el programa estadístico SPSS (IBM SPSS Statistics 25), para el análisis de los conjuntos de datos mediante procedimientos estadísticos avanzados. El uso de este ha facilitado la organización, jerarquización e interpretación de la información que devino de la búsqueda.

La relevancia de esta investigación radica en las aportaciones al análisis del tratamiento informativo y la conceptualización del problema en los medios digitales tomando a Cuba como contexto particular de análisis.

\subsection{Revisión teórica y planteamiento del problema}

\subsubsection{Del género a la violencia de género}

La concepción del género como producto de las relaciones culturales, tiene sus bases en el campo de la psiquiatría, utilizado para resolver problemas relativos a la identidad. Fue en la década de 1950 cuando John Money, especialista en el estudio de 'defectos' genitales congénitos, y sus colegas del Johns Hopkins Medical Center de Baltimore, EEUU desarrollaron una teoría del tratamiento psico-médico de los intersexos que da prioridad al condicionamiento socio-cultural de la identidad de género por encima del sexo biológico (Stolke, 2004, p. 87).

Luego sería acuñado por el antropólogo Gayle Rubin en 1975, quien define la categoría sexo-género como "un conjunto de disposiciones por el que una sociedad transforma la sexualidad biológica en productos humanos" (Rubin en Laurenzo, Maqueda \& Rubio, 2008, p. 53). Seyla Benahbib parte de esta definición y se acerca al carácter social de la categoría al definirlo como la constitución simbólica y la interpretación socio-histórica de las diferencias anatómicas entre los sexos (1990, p. 125).

Es frecuente que se equiparen las categorías sexo-género, de tal manera que en muchos discursos sociales se sustituye un término por el otro como si fueran lo mismo. "El género es un principio de orden, revela la existencia y los efectos de una relación de poder, de una diferencia, de un encuentro desigual" (Cirillo, 2005, p. 42). No sólo designa una clasificación para hombres y mujeres, sino que legitima una condición social para ambos y explica las esencias del sistema patriarcal.

La violencia contra las mujeres es una violencia estructural, que surge condicionada por las desigualdades impuestas del sistema patriarcal, "en cuyo origen está la división hombre/mujer, luego establece las raciales, económicas de clase de etnia, de edad, etc." (San Segundo, 2008, p. 67), por tanto, también la intensifican otras desigualdades sociales. 
Definir las bases de este problema ha sido objeto de múltiples campos interdisciplinarios. En la Convención de Belém do Pará (Brasil) se definió a la violencia contra las mujeres como "cualquier acción o conducta, basada en su género, que cause muerte, daño o sufrimiento físico, sexual o psicológico a la mujer, tanto en el ámbito público como en el privado" (Laurenzo, Maqueda \& Rubio, 2008, p. 63).

Las dificultades para conceptualizar a la violencia de género han determinado que el establecimiento de sus tipologías sea también limitada. En un Informe de la RAE sobre las diversas expresiones que se usan para expresar la violencia que es ejercida de los hombres contra las mujeres, quedan agrupadas las más frecuentemente usadas en idioma español. A continuación, se presentan los datos más significativos:

\section{Tabla 1}

Documentación de las diversas expresiones usadas en español para expresar el concepto.

\begin{tabular}{|l|l|l|l|}
\hline \multicolumn{1}{|c|}{ Términos } & $\begin{array}{c}\text { Documentación } \\
\text { Internet (Google) }\end{array}$ & Documentación CREA & \multicolumn{1}{|c|}{$\begin{array}{c}\text { Año Primera } \\
\text { Documentación } \\
\text { CREA }\end{array}$} \\
\hline Violencia doméstica & 100000 & $136(72)^{3}$ & 1983 \\
\hline Violencia intrafamiliar & 45000 & $49(34)$ & 1993 \\
\hline Violencia de género & 37700 & $19(9)$ & 1993 \\
\hline $\begin{array}{l}\text { Violencia contra las } \\
\text { mujeres }\end{array}$ & 35800 & $17(11)$ & 1977 \\
\hline Violencia familiar & 30000 & $34(25)$ & 1988 \\
\hline Violencia de pareja & 3000 & 1 & 2001 \\
\hline $\begin{array}{l}\text { Discriminación por } \\
\text { sexo }\end{array}$ & 13100 & 70 & 1983 \\
\hline
\end{tabular}

Fuente: Universitat de Valencia (https://www.uv.es/ivorra/documentos/Genero.htm)

En un intento por agrupar los términos que han sido referidos para designar el problema, quedan apuntados a continuación algunos de estos conceptos.

Violencia doméstica: hace referencia al espacio físico en que se produce, omite el entendimiento de quien es la víctima y su principal agresor, pues este ambiente puede sugerir múltiples víctimas y perpetradores de violencia. El Consejo de Europa define a la violencia doméstica como: "todos los actos de violencia física, sexual, psicológica o económica que se producen en la familia o en el hogar o entre cónyuges o parejas de hecho antiguos o actuales, independientemente de que el autor del delito comparta 0 haya compartido el mismo domicilio que la víctima" (2011, p. 5).

Violencia contra las mujeres: alude a la víctima de la violencia, pero no a su principal agresor, ya que puede tratarse de mujeres y de hombres. En el Convenio del Consejo 
de Europa sobre prevención y lucha contra la violencia contra las mujeres y la violencia doméstica se reconoce a la violencia contra las mujeres como "una violación de los derechos humanos y una forma de discriminación contra las mujeres, y designará todos los actos de violencia basados en el género que implican o pueden implicar para las mujeres daños o sufrimientos de naturaleza física, sexual, psicológica o económica, incluidas las amenazas de realizar dichos actos, la coacción o la privación arbitraria de libertad, en la vida pública o privada" (2011, p. 5).

Violencia masculina hacia las mujeres: es un término, aunque bastante similar al anteriormente citado, expresa fácticamente el proveedor de la violencia que sufren las mujeres. El uso de la preposición 'hacia' también le da un cambio al concepto, pues implica el sentido hacia el que se produce la violencia, pero le quita la gravedad que transfiere la preposición 'contra'. Autores como Luis Bonino la conceptualizan como "toda forma de coacción, control o imposición ilegítima por la cual se intenta mantener la jerarquía impuesta por la cultura sexista, forzándolas a hacer aquello que no quieren, que no hagan lo que quieren o se convenzan de que lo que les dice el hombre es lo que se debe hacer" $(2005$, p. 1$)$.

Violencia machista: para algunos autores este término connota más agresivamente la significación de la violencia y pone nombre a sus principales perpetradores. La fuerza de estos argumentos ha llevado a que autores como Concepción Fernández Villanueva se acoja al término y ponga énfasis en sus esencias, "se trata de actos motivados y permitidos por el machismo, por los valores machistas que persisten en la sociedad" (Yébenes en Peris, 2013, p. 184).

Violencia patriarcal o sexista: término utilizado para connotar la razón que da lugar a la violencia, especialmente dirigido a visibilizar el patriarcado y el sexismo social como argumento capital. Así lo entiende Norma Vázquez, al señalarlo más como un concepto analítico que descriptivo que pone énfasis en "el sexismo o machismo, como actitud de desprecio hacia lo femenino y las mujeres, y de reivindicación de la supremacía de lo masculino" (Vázquez, 2012, p. 10).

Terrorismo de género, sexual, machista: el término ha sido usado por algunos auto-res para otorgarle mayor fuerza a la connotación de la violencia de género con la intención de provocar el rechazo social que inspira el terrorismo. El acelerado boom global en contra de este ha promovido que se le asocien crímenes de diversa naturaleza. Carole Sheffield lo define como un sistema a través del cual los hombres atemorizan y, consiguientemente, controlan a las mujeres, el cual sirve, además, para recordar a las mujeres y a las niñas que ellas son vulnerables a las agresiones masculinas sólo por el hecho de ser mujeres (1993, p. 73).

El término también es defendido por Celia Amorós, al plantear que la resignificación del lenguaje es un medio para conseguir una serie de conceptualizaciones feministas 
que produzcan el efecto de politizar, contrarrestando, así, las conceptualizaciones patriarcales que intentan trivializar y despolitizar determinados fenómenos como la violencia machista (2008, pp. 17-20).

Violencias contra las mujeres (VVCMM): la autora Belén Zurbano propone pluralizar los términos violencia y mujer en el sentido de marcar la diversidad de formas que adopta esta violencia y significar las heterogéneas experiencias de mujeres. La definición da nombre a "los actos y amenazas de tales actos, perpetrados en la vida pública y en la privada, que supongan peligro para la vida, la integridad, el cuerpo y la dignidad de las mujeres y las niñas y cuyo origen (explícito o no) está en la voluntad de control y sometimiento del género femenino existiendo un modelo socio-ideológico subyacente que determina los roles, actitudes e identidades de los sujetos sociales (hombres y mujeres) según su condición sexual y en la que influyen igualmente otras categorías como la clase, la etnia, la religión y la heteronormatividad, entre otras" (2015, p.114).

Violencia de género: "La expresión 'violencia contra las mujeres' se refiere a todo acto de violencia basado en el género que tiene como resultado posible o real un daño físico, sexual o psicológico, incluidas las amenazas, la coerción o la privación arbitraria de la libertad, ya sea que ocurra en la vida pública o en la privada" (Naciones Unidas, 1995, p. 51-52).

Atendiendo a su naturaleza, al tipo de prácticas y a sus ámbitos, Zurbano propone una clasificación del término basada en el tipo de daño ejercido $(2015,134-136)$, de ahí a que considere clasificar estos elementos en distintas dimensiones: la manifestación violenta, que incluye la violencia física; violencia sexual; violencia psicológica; violencia económica; violencia simbólica. Otro elemento son las prácticas violentas, las que constituyen los modos en que se ejerce la violencia y se puede manifestar a través de palizas, amenaza, acoso, humillaciones, etc. Finalmente se encuentran los ámbitos de perpetración de dichas violencias, clasificados en domésticos, afectivos, socialescomunitarias, y también pueden estar a nivel estatal-institucional.

\subsubsection{Violencia de género en medios de comunicación}

En la práctica los medios de comunicación se han legitimado como instrumentos del sistema patriarcal. En el estudio sobre el tratamiento informativo que se le ha dado al problema de la violencia de género se han destacado algunos de los elementos que pueden estar influyendo en esta realidad. "Por una parte, los intereses económicos de los grandes grupos mediáticos que aseguran resultados elaborando relatos que apoyan y fortalecen los valores sociales dominantes sobre los cuales se asienta su negocio; en segundo lugar, la falta de sensibilidad y de conciencia social de la profesión periodística, asentada en la también falta de conocimiento y de formación sobre el tema, y por último, el tercer eje se situaría en los procesos y rutinas de producción que 
obliga a la profesión a reproducir a menudo las noticias de agencia, sin el tiempo necesario para contextualizar, documentarse, contrastar fuentes; en una palabra, para elaborar información de calidad" (López, 2002, p. 27).

Bajo la aparente pluralidad de la que dicen ser portadores, tanto en el sentido de la obsesión por el equilibrio de noticias buenas y malas y en el sentido ideológico, terminan presentando una realidad en algunos casos ficticia. "Como consecuencia de la acción de los periódicos, de la televisión y de los demás medios de comunicación, el público es consciente o ignora, presta atención o descuida, enfatiza o pasa por alto, elementos específicos de los escenarios públicos. La gente tiene a incluir o a excluir de sus propios conocimientos lo que los media incluyen o excluyen de su propio contenido. El público además tiende a asignar a lo que incluye una importancia que refleja el énfasis atribuido por los mass media a los acontecimientos, a los problemas, a las personas" (Shaw, 1979, p. 96), tesis que sostiene la Teoría de la Agenda-setting. Reconocer que lo que no muestran los medios es una elaboración de la realidad y no su reflejo o una ventana abierta al mundo, es la primera condición para poder diferenciar entre realidad y ficción. Si nos desarrollamos un espíritu crítico y un sentido de la búsqueda de la información alternativa a las vías formalmente establecidas, estamos condenados a la desinformación, a la capacidad para comprender nuestro mundo, y, por tanto, incapacitados para actuar con libertad (Serrano, 2009, p. 594).

Los medios digitales han constituido una fuente activa de información para los públicos. Gil de Zúñiga, Jung y Valenzuela (2012) refieren que sus usos van más allá de dar información, también contribuyen a fomentar los procesos democráticos y la creación de capital social.

Después de indagar en los resultados del estudio "La Prensa: digital vs papel", desarrollado por la Asociación para la Investigación de Medios de Comunicación (AIMC), cuyo trabajo de campo se realizó entre el 20 de mayo y el 16 de junio de 2011, Martín asegura que "con las nuevas herramientas se ha pasado de la lectura de noticias en papel a interactuar y participar de la información en tiempo real, accediendo a las noticias cuando están sucediendo. La información está al alcance de un click, deslocalizada y fragmentada" (2013, pp. 40-41). La facilidad de su acceso hace que también sea una preocupación atender el abordaje de los temas de género en estos espacios.

No se trata sólo de una mera revisión de las cuestiones de la subordinación femenina, tema que autoras como Simone de Beauvoir (1949), Gale Rubin (1975), Judith Butler (1990), Celia Amorós (1985), Amelia Valcárcel (1993, 1997, 2000) han tratado con suficiencia en sus textos, desde diferentes miradas, sino de analizar cómo esa discriminación se manifiesta de formas específicas, y tiene una representación mediática, reflejo a su vez de prácticas y estructuras validadas socialmente. 
El sensacionalismo y la especulación son mecanismos recurrentes en el uso que los medios le dan al tema. Muchos relatos han descansado en los reportes que dan organismos judiciales, por lo cual la información que se transmite se encuentra parcializada y sesgada con la opinión de solo una fuente. Se debe contrastar la información de forma que enriquezca el contenido y le de protagonismo a otros enfoques en su acercamiento al tema.

"De la misma forma que el periodismo de calidad exige especialización en otras áreas, trabajar con el tema de la violencia contra las mujeres no puede descansar solamente en "el sentido común" y en las experiencias personales de quienes trabajan con este tipo de información” (López, 2002, p. 27). La formación del personal de la prensa es un reto permanente puesto que son los productores del contenido y autores de los problemas a que se enfrenta el contenido.

\subsection{Planteamiento del problema}

La investigación bebe del análisis de contenido cualitativo de la información publicada en medios digitales en Cuba con énfasis en las noticias sobre violencia de género. Se determinó la muestra a partir de un muestreo no aleatorio subjetivo por decisión razonada, "en este caso las unidades de la muestra se eligen en función de algunas de sus características de manera racional y no casual" (Piccini, 2011, p. 56). Se han analizado aquellos textos periodísticos publicados en diversos medios de comunicación digitales que tienen a Cuba como foco de atención, con una temporalidad comprendida de enero a diciembre de 2017.

La selección del corpus en prensa digital estuvo determinada por elementos como la temática (contenidos sobre violencia de género en Cuba); el género periodístico (por la escasez de contenidos sobre casos específicos de violencia de género resultado de una primera búsqueda, se limitó el corpus a contenidos tanto de enfoque informativo como de opinión e interpretativo); período de tiempo: año 2017 (constituye un espacio de tiempo lo suficiente amplio como para estudiar la realidad más inmediata en torno al problema); tipos de medios: digitales (su acceso es viable para los fines de esta investigación, además, por la pluralidad de contenidos mediáticos que existen sobre Cuba en estos entornos) y estatales e independientes (oficiales y no oficiales, dentro de estos últimos se procedió a la distinción de opositores y no opositores, teniendo en cuenta la declaración de intereses de los medios seleccionados en sus Sitios Webs).

Para la selección del corpus se procedió a una búsqueda manual, dada la inexistencia de una hemeroteca virtual que archive los contenidos de los medios cubanos sobre violencia de género. En este proceso se utilizaron dos vías principales:

1. Consulta mediante el buscador interno de cada medio, lo que se vio dificultado por la existencia de buscadores básicos por palabras claves que no permitían una bús- 
queda avanzada por período de tiempo. Las palabras claves empleadas para la búsqueda fueron: 'violencia mujer Cuba', 'violencia de género Cuba' 'asesinato mujer Cuba' y 'muerte mujer Cuba'.

2. Se utilizó el buscador de Google como vía complementaria dada la escasez de textos que devolvieron los buscadores internos de los medios seleccionados y con el fin de garantizar que el corpus estuviera completo. La elección no fue aleatoria puesto que Google está reconocido mundialmente como el motor de búsqueda más completo y eficiente en la búsqueda de contenidos en Internet. Esta herramienta le aporta gran parte del tráfico web a los medios ya que los usuarios suelen alcanzar los contenidos que necesitan como resultado de la navegación indirecta a través de este motor. Aunque le han sido identificados diversos sesgos e irregularidades en la recogida de información y organización de las búsquedas, por ejemplo, el hecho de que pondera el posicionamiento de algunos medios frente a otros, continúa siendo un referente esencial en el estudio de contenidos en la Web.

El corpus determinando es lo suficientemente amplio como para considerarse relevante. Con su determinación se intenta estudiar una parte de la realidad en torno al tratamiento de la violencia de género en la prensa digital, profundizando en la conceptualización que hacen del término. Estuvo conformado por 86 textos seleccionados de un total de 15 medios de comunicación digitales, dentro de los que fueron contemplados 7 medios oficiales que son cabecera nacional de la prensa en Cuba (Granma, Juventud Rebelde, Cubadebate, Cubasi, Trabajadores, ACN, Cubahora). Por otro lado, también fueron tomados medios opositores y no opositores cubanos, con el ánimo de enriquecer el corpus y de distinguir elementos diferenciales en el abordaje que hacen los medios de distinto perfil sobre la violencia de género y su conceptualización. Para ello se seleccionaron también 3 medios de propiedad no estatal no opositores, algunos de los que han sido financiados por cubanos radicados en el exterior y otros que tienen convenio con el gobierno (Progreso Semanal, OnCuba, Cubaposible) y 5 opositores (14 y Medio, Cubanet, Diario de Cuba, CiberCuba, Martí Noticias).

La siguiente tabla ilustra la descripción de los medios digitales de los que se seleccionó el corpus, atendiendo a los géneros periodísticos y número de textos encontrados. 
Tabla 2

Total de textos periodísticas publicadas en 2017 por los medios objeto de estudio.

\begin{tabular}{|c|c|c|}
\hline Nombre del medio & Géneros periodísticos & No. de textos \\
\hline \multicolumn{3}{|c|}{ Medios de propiedad estatal (Oficiales) } \\
\hline Granma Internacional & $\begin{array}{l}\text { Noticias: } 6 \\
\text { Entrevistas de perfil: } 1 \\
\text { Artículos: } 2 \\
\text { Reportajes objetivos: } 1 \\
\text { Entrevista de } \\
\text { declaraciones: } 1\end{array}$ & 11 \\
\hline Juventud Rebelde & $\begin{array}{l}\text { Noticias: } 3 \\
\text { Entrevista de perfil: } 1 \\
\text { Columna: } 1 \\
\text { Reportaje objetivo: } 2 \\
\text { Artículo: } 1\end{array}$ & 8 \\
\hline Trabajadores & Noticias: 3 & 3 \\
\hline Cubadebate & $\begin{array}{l}\text { Noticias: } 3 \\
\text { Entrevista de } \\
\text { declaraciones: } 2 \\
\text { Reportaje objetivo: } 1\end{array}$ & 6 \\
\hline Cubasí & Reportaje objetivo: 1 & 1 \\
\hline Agencia Cubana de Noticias (ACN) & Noticias: 3 & 3 \\
\hline Cubahora & Artículo: 2 & 2 \\
\hline \multicolumn{3}{|c|}{ Medios de propiedad no estatal (Independientes) } \\
\hline Progreso Semanal & $\begin{array}{l}\text { Noticia: } 2 \\
\text { Crónica: } 1 \\
\text { Artículo: } 1 \\
\text { Reportaje objetivo: } 1\end{array}$ & 5 \\
\hline OnCuba Web & $\begin{array}{l}\text { Crónica: } 2 \\
\text { Columnas: } 3 \\
\text { Reportaje objetivo: } 1\end{array}$ & 6 \\
\hline Cubaposible & Crítica: 2 & 2 \\
\hline Martí Noticias & $\begin{array}{l}\text { Noticia: } 1 \\
\text { Reportajes objetivos: } 5\end{array}$ & 6 \\
\hline 14 y Medio & $\begin{array}{l}\text { Noticias: } 2 \\
\text { Reportaje objetivo: } 1 \\
\text { Columna: } 1\end{array}$ & 4 \\
\hline
\end{tabular}


Tabla 2 (Continuación)

\begin{tabular}{|l|l|l|}
\hline \multicolumn{1}{|c|}{ Nombre del medio } & Géneros periodísticos & No. de textos \\
\hline Cubanet & $\begin{array}{l}\text { Noticias: } 8 \\
\text { Artículo: } 1 \\
\text { Reportaje objetivo: } 1 \\
\text { Entrevista de } \\
\text { declaraciones: } 1\end{array}$ & 11 \\
\hline CiberCuba & $\begin{array}{l}\text { Noticias: } 8 \\
\text { Artículo: } 1\end{array}$ & 9 \\
\hline Diario de Cuba & $\begin{array}{l}\text { Noticias: } 6 \\
\text { Reportaje objetivo: } 2 \\
\text { Artículo: } 1\end{array}$ & 9 \\
\hline
\end{tabular}

Fuente: Elaboración propia

La determinación de los elementos que conforman la ficha de análisis se toma de propuestas validadas en investigaciones sobre el tratamiento informativo de la violencia de género. En este sentido fue oportuno remitirnos a la Tesis Doctoral de la Dra. Belén Zurbano Berenguer (Zurbano, 2015), quien determina categorías de análisis para estudiar la construcción mediática de la violencia de género. También se bebe de la ficha de A. Carratalá en su estudio sobre tratamiento informativo de casos de violencia en parejas del mismo sexo (2016); así como también de las autoras Daniela Aguilar Freire y Claudia Rodríguez Hidalgo en su estudio sobre el feminicidio en la prensa ecuatoriana (2018).

Esta investigación se nutre de las aportaciones de José Manuel Zorrilla Barroso en su tesis doctoral (1996), del que tomamos los elementos para estudiar el titular del texto periodístico, necesario para conocer más a fondo el tratamiento informativo del tema.

Por tanto, la ficha de análisis comprende categorías descriptivas del texto periodístico (género, exclusividad, autoría, sección, recursos visuales, tipo de titular); otras más relacionadas con el contenido (fuentes informativas consultadas, vías de prevención y ayuda, uso de estadísticas, referencia a la legislación) y con la forma en que se conceptualiza el problema (identificación nominal, enfoques periodísticos, ámbitos, formas de expresión).

\subsection{Resultados}

El análisis ha posibilitado un acercamiento al tratamiento de la violencia de género en la prensa digital oficialista y no oficialista cubana, así también han puesto foco en la 
conceptualización mediática que hacen los medios de comunicación digitales sobre la violencia de género.

Tabla 3

Descripción del Corpus de acuerdo a tipos de medios, número de textos y géneros periodísticos.

\begin{tabular}{|c|l|l|l|l|}
\hline & \multicolumn{1}{|c|}{$\begin{array}{c}\text { Medios } \\
\text { oficiales }\end{array}$} & $\begin{array}{c}\text { Medios no } \\
\text { opositores }\end{array}$ & $\begin{array}{c}\text { Medios } \\
\text { opositores }\end{array}$ \\
\hline No. de textos & & 34 textos & 13 textos & 39 textos \\
\hline $\begin{array}{c}\text { Cantidad de } \\
\text { textos por } \\
\text { géneros } \\
\text { periodísticos }\end{array}$ & Noticias & 18 & 2 & 24 \\
\cline { 2 - 5 } & $\begin{array}{l}\text { Entrevistas de } \\
\text { declaraciones }\end{array}$ & 2 & - & 1 \\
\cline { 2 - 5 } & Reportajes objetivos & 5 & 2 & 10 \\
\cline { 2 - 5 } & Artículos & 5 & 1 & 3 \\
\cline { 2 - 5 } & Crítica & - & 2 & - \\
\cline { 2 - 5 } & Columna & 1 & 4 & - \\
\cline { 2 - 5 } & Crónicas & - & 2 & - \\
\cline { 2 - 5 } & Entrevistas de perfil & 3 & - & - \\
\hline
\end{tabular}

Fuente: Elaboración propia

Existe carencia de una agenda sistemática en el abordaje de la violencia de género. En la Figura 1 se muestra la fluctuación constante que evidencia la inestabilidad que ocupa el tema en las agendas de los medios digitales seleccionados. Se puede ver que diciembre es el mes de mayor concentración de textos, lo cual se corresponde con la importancia que adquiere mediáticamente por la celebración de la Jornada internacional de lucha por la eliminación de la violencia contra las mujeres. Esta jornada se celebra en Cuba durante 16 días de activismo social, periodo en el que los medios se encargan de darle mayor cobertura al problema mediante las acciones que fomenta la jornada. De manera general se trata de contenidos noticiosos sobre lo que va transcurriendo. 


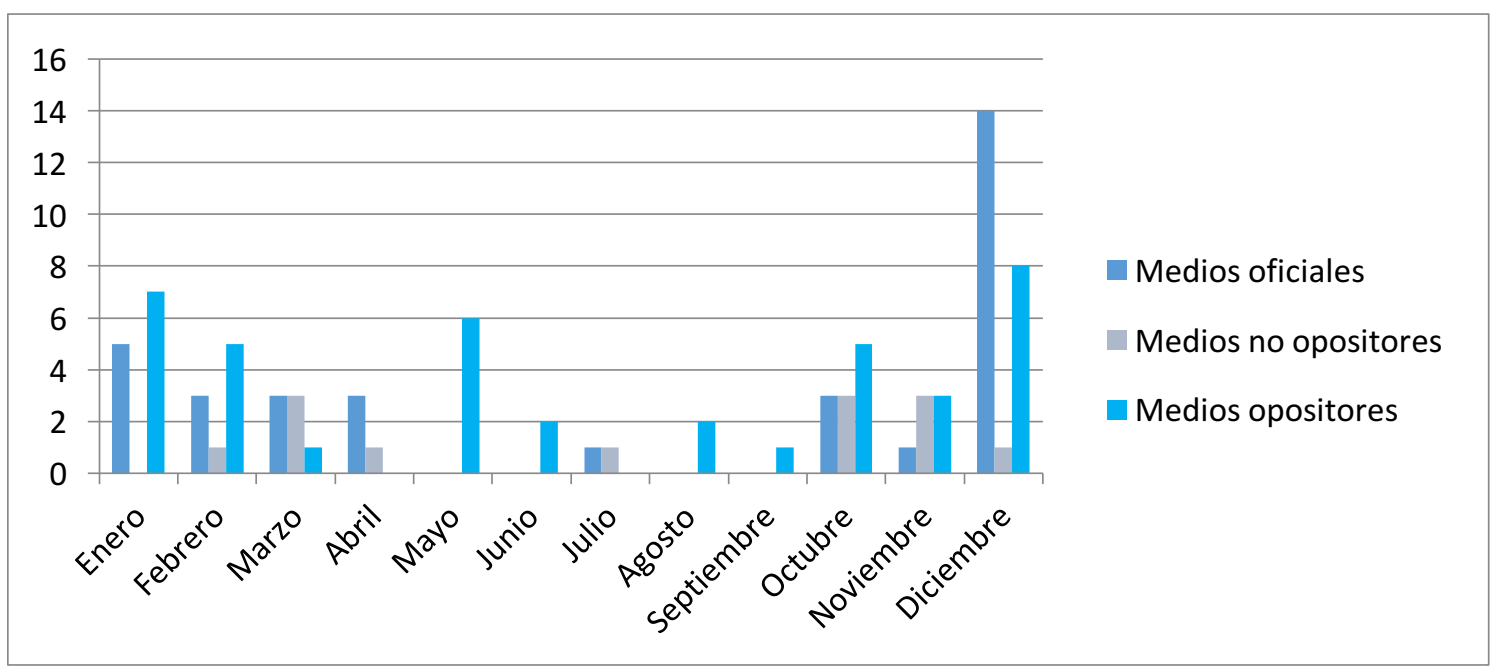

Figura 1. Distribución en cantidad de noticias que tuvo el tema de la violencia de género de enero a diciembre de 2017 en los tipos de medios determinados.

Fuente: Elaboración propia

\subsubsection{Definiciones que sobre violencia de género se han producido en el ámbito mediático digital en el contexto de Cuba}

De acuerdo a las definiciones que sobre violencia de género se han producido en el ámbito mediático digital en el contexto de Cuba, se puede ver que la violencia de género es reconocida bajo variedad de términos, apreciándose en cada uno de los tipos de medios una determinada recurrencia a nombrarlo como violencia doméstica, en el caso de los medios opositores cuando hacen alusión a casos específicos. El uso del término 'violencia (contra/hacia/a) la mujer es más asiduo en medios oficiales, los que emplean indistintamente la preposición, otorgándole la misma connotación y fuerza cuando en realidad no la tienen. La mención terminológica los medios no opositores seleccionados divaga entre la utilización de varios términos. En los contenidos no se aprecia asociado al término una definición que paute lo que se entiende por el problema en las diversas formas en las que se encuentra planteado.

El concepto 'violencia de género' abarca otros delitos hacia la mujer como la prostitución forzada, los crímenes de honor o la mutilación genital, la agresión sexual o la trata de mujeres con fines de explotación sexual. Son delitos mayoritariamente dirigidos a la mujer por el hecho de serlo. Sin embargo, en muchos textos tienden a independizarse como problemas independientes. 
Tabla 4

Frecuencia de terminología empleada según tipos de medios.

\begin{tabular}{|l|l|l|l|}
\hline & Medios oficiales & $\begin{array}{c}\text { Medios no } \\
\text { opositores }\end{array}$ & Medios opositores \\
\hline $\begin{array}{l}\text { Sin terminología de } \\
\text { referencia }\end{array}$ & 3 & 2 & 5 \\
\hline Violencia de género & 10 & 2 & 9 \\
\hline Maltrato & - & 1 & - \\
\hline Violencia doméstica & 3 & - & - \\
\hline $\begin{array}{l}\text { Violencia } \\
\text { (contra/hacia/a) la } \\
\text { mujer }\end{array}$ & 7 & 1 & 2 \\
\hline Feminicidio & - & 1 & 1 \\
\hline Varios términos & 11 & 4 & 20 \\
\hline Otras referencias & - & 2 & 2 \\
\hline
\end{tabular}

Fuente: Elaboración propia

De manera general se percibe una alta tendencia de no hacer referencias a prácticas concretas de violencia de género. Los enfoques centrados en agresiones se utilizan en la mayor parte para hacer referencia a casos específicos y la narración de estos es en extremo escasa, sobre todo en las agendas de medios oficiales.

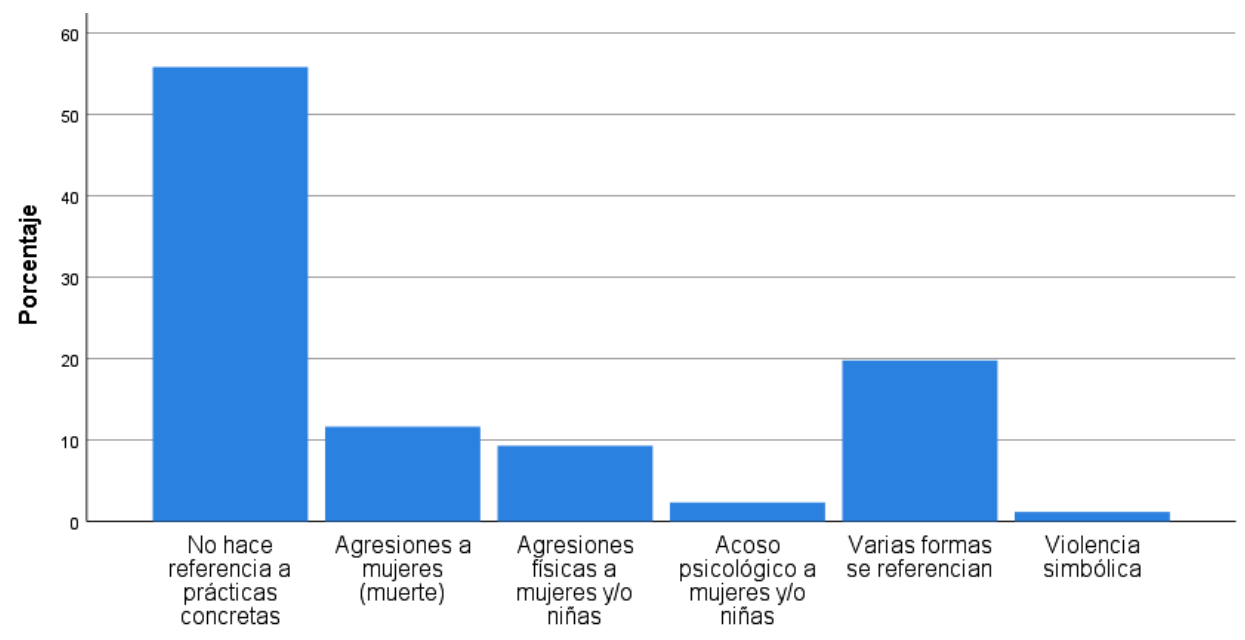

Figura 2. Frecuencia porcentual de los enfoques periodísticos de los textos sobre violencia de género.

Fuente: Elaboración propia

Los medios oficiales tienden a hacer más alusión al ámbito social comunitario en un intento por llamar la atención sobre las formas de expresión de la violencia de género 
que ocurren en estos espacios, mientras que los opositores amplían más en los espacios domésticos. Los contenidos producidos por los medios no oficiales seleccionados son muy variables en cuanto a la referencia de ámbitos específicos, apoyándose en su mayoría en géneros periodísticos de opinión e interpretación se acercan al tema desde la pronunciación de varios ámbitos y formas de expresión.

Tabla 5

Frecuencia de ámbitos de la violencia de género mencionados por tipos de medios.

\begin{tabular}{|l|l|l|l|}
\hline & \multicolumn{1}{|c|}{ Medios oficiales } & \multicolumn{1}{|c|}{$\begin{array}{c}\text { Medios no } \\
\text { opositores }\end{array}$} & \multicolumn{1}{|c|}{$\begin{array}{c}\text { Medios } \\
\text { opositores }\end{array}$} \\
\hline Doméstico & 2 & - & 10 \\
\hline Afectivo & - & 2 & 7 \\
\hline Social-Comunitario & 12 & 1 & 9 \\
\hline Estatal & - & - & 2 \\
\hline $\begin{array}{l}\text { Varios ámbitos en } \\
\text { el mismo nivel }\end{array}$ & 8 & 7 & 5 \\
\hline No hay referencia & 13 & 3 & 5 \\
\hline
\end{tabular}

Fuente: Elaboración propia.

Las formas de expresión son diversas en los textos, siendo menores los casos en los que se aprecia un solo tipo de manifestación. La no mención de las formas de expresión del problema se presenta en un $46,5 \%$, siendo la más frecuente práctica en la producción de los contenidos. En $23,3 \%$ se mencionan varias prácticas y en un $18,6 \%$ se muestran otras. Los indicadores relativos a formas concretas son inferiores, siendo el menos empleado la intimidación sexual $(1,2 \%)$.

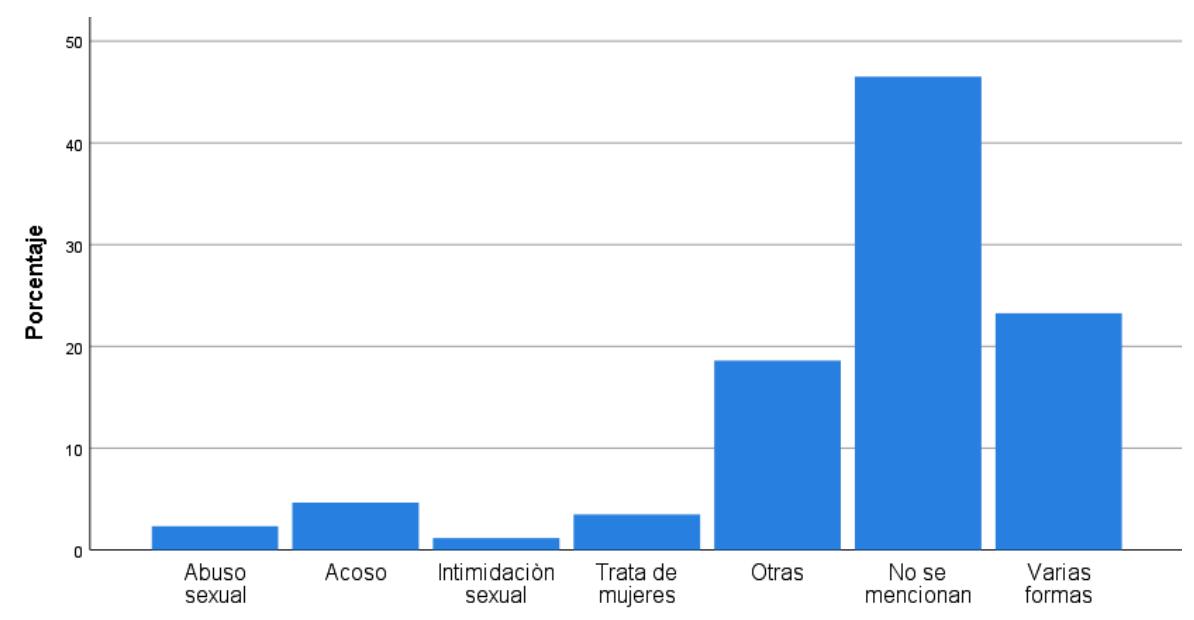

Figura 3. Frecuencia porcentual de formas de expresión de la violencia de género que se presentan en los textos.

Fuente: Elaboración propia. 
1.3.2. Caracterización del tratamiento de los contenidos sobre violencia de género en Cuba en los medios digitales seleccionados

Se observa poca sistematicidad de los textos sobre violencia de género en Cuba durante todo el año. Los contenidos recurren a una excesiva institucionalización que bebe en su mayoría de fuentes expertas en el caso de medios oficiales y fuentes de diverso tipo en el caso de medios no oficiales. La referencia al tema tiende a hacerse ante acontecimientos y fechas que ponen el alza el problema. Es mayor la atención que se le presta a la efeméride o al acontecimiento, manifestándose en la mayor parte de los casos un tratamiento anecdótico que no profundiza.

De manera general hay una tendencia en la utilización de géneros informativos, donde predomina la noticia $(51,2 \%)$ y el reportaje objetivo $(19,8 \%)$. Mientras que de los géneros de opinión y de interpretación su porcentaje es menor en comparación con los anteriores, ocupando los mayores niveles, el artículo con un 10,5\% y la entrevista de perfil con un $3,5 \%$

La referencia a la legislación y las estadísticas son escasas. Cerca del $90 \%$ de los recursos visuales empleados son imágenes. La mayor parte de los textos son exclusivos del medio donde se publica, sólo en 9 ocasiones se toman contenidos de otros. La autoría de los textos se identificó un $95,3 \%$ de las veces. La visibilidad que se le da al autor de la noticia "puede valer como indicador de la calidad de un diario, dado que la carencia de una autoría clara en las noticias difundidas por el medio, lo despersonaliza, a la vez que enfría la noticia, provocando que el lector vea el suceso narrado como algo no ya objetivo, sino impersonal, de lo que puede o no fiarse, porque no sabe quién se lo está contando" (López \& Domínguez, 2012, p. 792).

Otro elemento característico en su abordaje es el enfoque hacia las vías de prevención. En el $14 \%$ de los textos se mencionan vías de prevención a la violencia de género. La escasa mención que se hace no garantiza toda la información necesaria que deban tener como ayuda las personas víctimas de la violencia de género. La referencia a la legislación adolece de un enfoque crítico en torno a las carencias que presenta la vigente en la prevención y tratamiento de los casos de violencia de género. Las citas en la prensa oficialista abundan de un excesivo triunfalismo en torno a la normativa, reconociendo sólo en algunas ocasiones la necesidad de trabajar en su perfeccionamiento.

Los medios oficiales tendieron a ubicar la noticia con mayor frecuencia en las secciones de 'Cuba' y 'Sociedad' y esto lo explica el carácter institucional que ocupa la mayor parte de los textos, enfocados en su mayoría en la cobertura sobre eventos relacionados con la temática. En el caso de los medios no opositores hay una mayor tendencia a ubicarlas dentro de otras secciones no especificadas. Los medios opositores 
destacan por prevalecer con contenidos noticiosos ubicados en su mayoría en las secciones de 'Cuba' y 'Noticias'.

Se aprecia una mayor frecuencia de utilización de titulares designativos, evocadores y temáticos, mientras que en menor medida son empleados los apelativos. Una visión global de esto nos dice que los textos suelen producirse bajo titulares enfocados en la noticiabilidad de acontecimientos. Ejemplos:

Texto tomado de Juventud Rebelde (20/10/2017)- "Cienfuegos: El caso de Leidy Maura Pacheco Mur".

Texto tomado de Cubanet (21/02/2017) "Crimen pasional: Mujer es asesinada a puñaladas en Arroyo Naranjo".

\subsection{Discusión y conclusiones}

En Cuba la producción periodística sobre violencia de género en los medios digitales oficiales encuentra muy limitada cobertura. Desde los medios digitales no ha existido una coherencia informativa en la manera de abordar este problema. La inespecificidad del término ha estado condicionado entre otras cosas por la ausencia de una legislación que paute la conceptualización del problema y por la ausencia de una normativa que exija el debido tratamiento a las agendas mediáticas digitales. Estos elementos permiten ratificar la comprobación de la hipótesis planteada.

El camino hacia el cambio y la educación del sector periodístico en materia de violencia de género pasa por la sensibilización de los profesionales que laboran en este sector sobre el tema, una de las más asentadas barreras que dificulta esta tarea es la invisibilidad que ocupa y cuando se aborda no siempre se hace con la profundidad requerida. La baja prioridad que se le otorga ha influido en que los temas vinculados con el fenómeno se ocupen solo para llenar agendas informativas o cuando se celebra alguna fecha que recuerde que la violencia de género es un problema social de amplias dimensiones.

Las conclusiones de esta investigación tienen un carácter descriptivo y limitado. Dado los cambios recientes que han tenido lugar en la política del país, sería pertinente un análisis más extendido en el tiempo y que abarque la actualidad relativa al tema. Constituye un imperativo a día de hoy enfocarnos en los consumidores de la información de los medios. Por tanto, la investigación también abre aristas que pudieran ser de interés en el estudio de los consumidores de la información de los medios pues conocer sus percepciones sobre violencia de género dentro del contexto cubano es una necesidad para adecuar los contenidos dirigidos a los mismos. 


\section{Notas}

${ }^{1}$ Femicidios: Marcela Lagarde lo propone como 'feminicidio'. Según la autora, femicidio sólo significa el homicidio de mujeres y oculta la verdadera implicación social del asesinato de mujeres por parte de los hombres, de ahí a que proponga como voz de mayores connotaciones el "feminicidio", calificándolo como "conjunto de violaciones a los derechos humanos de las mujeres que contienen los crímenes y las desapariciones de las mujeres (...) es el genocidio contra mujeres y sucede cuando las condiciones históricas generan prácticas sociales que permiten atentados violentos contra la integridad, la salud, las libertades y la vida de niñas y mujeres" (Lagarde, 2008, p. 216).

${ }^{2}$ CREA (Corpus de referencia del Español Actual).

${ }^{3}$ Número de casos y, entre paréntesis, número de documentos.

\section{Referencias}

Aguilar, D \& Rodríguez, C. (2018). El femicidio en la prensa ecuatoriana: análisis de contenido de los diarios El Universo y El Comercio. Estudios sobre el Mensaje Periodístico No. 24, pp. 13-32.

Alonso, Z. K. (28 de julio de 2018). Violencia de género en los medios de comunicación. Resumen latinoamericano. Recuperado de: http://www.resumenlatinoamericano.org/2018/07/28/violencia-de-genero-en-los-mediosde-comunicacion/

Amorós, C. (1985). Crítica de la razón patriarcal. Barcelona: Anthropos.

(2005). Dimensiones de poder en la teoría feminista. Revista Internacional de Filosofía Política, No. 25, pp. 11-34.

(2008). Conceptualizar es politizar. En: Patricia Laurenzo et al. (coords.): Género, violencia y derecho. Tirant lo Blanch, Valencia, pp. 15-25.

Beauvoir, S. (2007). El segundo sexo (1949). Trad. de Juan García Puente. Buenos Aires: Sudamericana.

Benhabib, S. (1990). El otro generalizado y el otro concreto: controversia Kolhberg-Gilligan y la teoría feminista, en Seyla Benhabib y Drucilla Cornell (Eds.), Teoría feminista y teo-ría crítica, Alfons el Magnànim, Valencia.

Bonino, L. (2005). La violencia masculina contra la pareja. Edición Museo Nacional de Arte Reina Sofía. Recuperado en https://bit.ly/2OqD0lc.

Butler, J. (1990). Gender Trouble: Feminism and the Subversion of Identity. Routledge: New York.

Carratalá, A. (2016). La información en prensa española sobre casos de violencia en parejas del mismo sexo. Revista Latina de Comunicación Social, No. 71, pp. 40-65. 
Cirillo, L. (2005). Virtualidades pedagógicas del feminismo para la izquierda, Revista Internacional de Filosofía Política (UNED-Madrid/UAM-México), n. 25.

Código Penal de Cuba. Ley No. 62, (29 de diciembre de 1987). Ministerio de Justicia. La Habana.

Consejo de Europa (2011). Convenio de Estambul del Consejo de Europa sobre prevención y lucha contra la violencia contra las mujeres y la violencia doméstica. Council of Europe Treaty Series, No. 210, Estambul. Recuperado en https://bit.ly/2M9uAs3.

Constitución de la República de Cuba (2019). Recuperado de: http://media.cubadebate.cu/wpcontent/uploads/2019/04/Constituci\%C3\%B3n-de-la-Rep\%C3\%BAblica-de-Cuba.pdf

Cuba. Informe nacional sobre la implementación de la agenda 2030 (2019). Recuperado de https://foroalc2030.cepal.org/2019/sites/foro2019/files/informe_nacional_voluntario_de_ cuba_sobre_implementacion_de_la_agenda_2030.pdf

Diz, G. E. (2011). Reubicar el futuro. Las mediaciones entre la cultura profesional y las lógicas de producción periodística para la web. Estudio de casos: Granma y Trabajadores (Tesis Doctoral). Universidad de La Habana, La Habana, Cuba.

García, J. L. (2013). Revolución, socialismo, periodismo. La prensa y los periodistas cubanos ante el siglo XXI. Editorial Pablo de la Torriente, Cuba.

Gayle, R. (1975). The Tra ffic in Women », Rayna R. Reiter (ed.), en Toward an Anthropology of Women. New York, Monthly Review Press.

Gil de Zúñiga, H.; Jung, N.; Valenzuela, S. (2012). Social media use for news and individuas's social capital, civic engagement and political participation. Journal of ComputerMediated Communication, 17, pp. 319-336. doi:10.1111/j.1083-6101.2012.01574.x

Informe del Comité Especial Plenario del vigésimo tercer período extraordinario de sesiones de la Asamblea General (2000). Naciones Unidas. No. 3 (A/S-23/10/Rev.1). Recuperado en https://bit.ly/2OXDoPI.

Lagarde, M. (2008). Antropología, feminismo y política: violencia feminicida y derechos humanos de las mujeres. México: Universidad Autónoma de México (UNAM).

Laurenzo, P.; Maqueda, M. L. \& Rubio, A. (2008). Género, violencia y derecho, Editorial Tirant lo Blanch, Valencia.

López, H. M. A. \& Domínguez, D. R. (2012). El valor documental del periodista como autor. Estudios sobre el Mensaje Periodístico. Vol. 18 Núm. 2 (2012), pp. 791-803.

López, P. (2002). La violencia contra las mujeres en los medios de comunicación. En Instituto Oficial de Radio Televisión (IORTVE) (Ed.), Mujer violencia y medios de comunicación, pp. 21-36. Recuperado en https://bit.ly/2OYZaCz. 
Martín, M. V. (2013). La eficacia de internet como medio de comunicación en el sector turístico español: el branding en los web turísticos. Tesis Doctoral. Universidad Complutense de Madrid.

Más, S. (7 de febrero de 2019). Cuba: Las leyes deben visibilizar el delito de violencia de género. AmecoPress. Recuperado de: http://www.amecopress.net/spip.php?article18908.

Moya, I. (2010). El sexo de los ángeles. Una mirada de género a los medios de comunicación. Centro Félix Varela, La Habana, Cuba.

Naciones Unidas (1995). Informe de la IV Conferencia Mundial sobre las Mujeres, Beijing.

Peris, M. (2013). La despolitización de la violencia de género a través de la terminología. Revista Asparkía, No. 24, pp. 176-194.

Piccini, J. (2010). Vigencia y actualización de la valoración documental. Universidad de la república, México D.F.

Piñuel, J. L. (2002). Epistemología, metodología y técnicas del análisis de contenido. Departamento de Sociología IV, Facultad de CC. de la Información Universidad Complutense de Madrid, Estudios de Sociolingüística 3 (1), España.

Proveyer, C. et al (2005). Selección de Lecturas de Sociología y Política Social de Género. La Habana: Editorial Félix Varela.

(2014). Violencia de género. Aproximación desde la realidad cubana. Revista Sexología y Sociedad. 20(1).

Proveyer, C. (2014). Violencia de género. Aproximación desde la realidad cubana. Revista Sexología Y Sociedad, 20(1). Recuperado de http://revsexologiaysociedad.sld.cu/index.php/sexologiaysociedad/article/view/465/507

San Segundo, M. T. (2008). Violencia de género. Una visión multidisciplinar. Editorial Universitaria Ramón Areces, Madrid.

Serrano, P. (2009). Desinformación. Cómo los medios ocultan el mundo, Ediciones Pe-nínsula, Barcelona.

Shaw, E. (1979). Agenda-setting and mass communication theory, en Gazette International Journal for Mass Communication Studies, vol. XXV, $n^{\circ} 2$.

Sheffield, C. (1993). The Invisible Intruder: Women's Experiences of Obscene Phone Calls en Pauline B. Bart y Eileen Geil Moran (eds.). Violence Against Women: The Bloody Footprints, Londres, Sage Publications, pp.73-78. 
Stolke, V. (2004). La mujer es puro cuento: la cultura del género. Estudios Feministas, Florianópolis, 12(2), pp. 77-10.

Tubert, S. (ed.) (2003). Del sexo al género. Los equívocos de un concepto. Cátedra, Universitat de València, Instituto de la Mujer, Madrid.

Valcárcel, A. (1993). Del miedo a la igualdad, Barcelona, Crítica.

(1997). La política de las mujeres, Madrid, Cátedra: Universidad de Valencia: Instituto de la mujer.

(eds.) (2000). Los desafíos del feminismo ante el siglo XXI. Hypatia, Instituto Andaluz de la Mujer.

Vázquez, N. (2012). Desigualdad de género y violencia sexista: un problema ineludible. Editorial Manu Robles-Arangiz Institutua, Bilbao. Recuperado el 5 de mayo de 2018 en https://bit.ly/2vU2WEr.

Zorrilla, J. M. (1996). El titular de la noticia. Estudio de los titulares informativos en los diarios de difusión nacional. Tesis Doctoral, Departamento de Periodismo 1, Universidad Complutense de Madrid.

Zurbano, B. (2015). Discurso periodístico y violencias contra las mujeres. Aproximaciones a la construcción y valoración de los mensajes informativos en la prensa de referencia en España (2000-2012). (Tesis Doctoral), Universidad de Sevilla, Sevilla, España.

Zurbano, B. \& García, M. (2016). Comunicación periodística y violencias contra las mujeres. Una propuesta metodológica para su abordaje integral. Revista Ámbitos, No. 34, pp. 113, Universidad de Sevilla, España.

Zurbano, B., \& García, M. (2017). Propuesta metodológica para la evaluación de la calidad deontológica de las noticias sobre violencias contra las mujeres. Communication \& Society, 30 (1), pp. 73-85. 


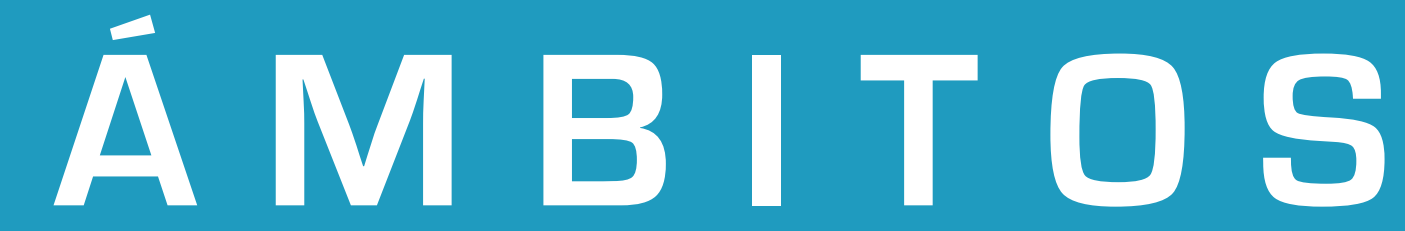

ISSN: 1139-1979 | ISSN digital: 1988-5733 | Depósito Legal: SE-1493-98

Revista Internacional de Comunicación editada por el Grupo de Investigación en Estructura, Historia y Contenidos de la Comunicación(GREHCCO) de la Universidad de Sevilla.

\section{ambitoscomunicacion@us.es}

http://institucional.us.es/ambitos

@RevistaAmbitos 